\title{
Spontaneous Hemopericardium as an Adverse Effect of Rivarox- aban Administration
}

\author{
Asif Surani, MD*, Beatriz Martinez Quintero, MD, David Brual, MD, Hadi Elias, MD, Fahad Uddin \\ Ahmed, MD and Matthew Peters, MD
}

Saint Agnes Hospital, 900 S Caton Ave, Baltimore, 21229, Maryland, USA

*Corresponding author: Asif Surani, MD, Saint Agnes Hospital, 3300 S Stonegate Circle, Apt 208, New Berlin, WI 53151, USA, Tel: 6672146641

\begin{abstract}
Rivaroxaban is a direct oral anticoagulant with a favorable safety profile when compared to warfarin in respect to bleeding. During post-marketing surveillance, intracranial and retroperitoneal bleedings have been reported; however, hemopericardium has not been frequently described. In this case series, we present three cases of spontaneous hemopericardium where rivaroxaban was thought to be the causative agent. Predisposing factors for rivaroxaban induced hemopericardium might include impaired kidney function, concomitant use of antiplatelet agents, herbal supplements, and CYP3A4 metabolizers and inhibitors. Clinicians should be aware of the possibility of such life-threatening adverse event in patients taking rivaroxaban and should consider it in their differential diagnosis in a patient presenting with chest pain and/or dyspnea while on rivaroxaban. This case series suggests that rivaroxaban induced hemopericardium might not be as rare as initially thought.
\end{abstract}

\section{Introduction}

Rivaroxaban, a factor Xa inhibitor, has been approved by the Food and Drug Administration (FDA) to be used as an anticoagulant in multiple medical conditions, such as deep venous thrombosis (DVT), DVT prophylaxis after knee and hip replacement, prevention of thromboembolism in nonvalvular atrial fibrillation, and pulmonary embolism. In recent years, several studies, including a meta-analysis of rivaroxaban and bleeding risks when compared to vitamin $\mathrm{K}$ antagonists, have shown that rivaroxaban has a favorable bleeding-related safety profile with a decrease in fatal bleeding, no suggestion of an increase in all-cause mortality, and no statistical significant difference when compared to vitamin $\mathrm{K}$ antagonists in the proportion of major and clinically relevant non-major bleeding [1]. Nonetheless, major bleeding events have been reported, including intracranial hemorrhage, gastrointestinal bleeding, retroperitoneal bleeding, and intraocular bleeding [2]. Yet, there is less than a handful of case reports describing spontaneous hemopericardium after administration of rivaroxaban [3-9]. We present a case series including three patients presenting with isolated spontaneous hemopericardium while receiving rivaroxaban.

\section{Case Report}

\section{Case 1}

A 66-year-old African American male with a past medical history of hypertension, type $2 \mathrm{DM}$, peripheral vascular disease status post right above knee amputation, and recent DVT on rivaroxaban presented with persistent chest pain for two weeks duration. The pain was worsened with lying down and taking deep inspiratory breaths, and was relieved slightly by bending forward. It was associated with dyspnea, diaphoresis, subjective fevers, decreased appetite and generalized weakness. His medications included rivaroxaban $20 \mathrm{mg}$ daily, gabapentin, glipizide, and losartan. Of note, the patient was admitted with similar chest pain ten days prior; workup at that time revealed ST elevation in anterolateral leads, however his cardiac catheterization and echocardiogram (ECHO) were unremarkable. On physical exam he was febrile with a temperature of 103.2 ${ }^{\circ} \mathrm{F}$ and tachycardic. Other significant findings included distant heart sounds, weak peripheral pulses, and pul-

Citation: Surani A, Quintero BM, Brual D, Elias H, Ahmed FU, et al. (2019) Spontaneous Hemopericardium as an Adverse Effect of Rivaroxaban Administration. Int J Clin Cardiol 6:161. doi. org/10.23937/2378-2951/1410161

Accepted: November 23, 2019; Published: November 25, 2019

Copyright: (C) 2019 Surani A, et al. This is an open-access article distributed under the terms of the Creative Commons Attribution License, which permits unrestricted use, distribution, and reproduction in any medium, provided the original author and source are credited. 


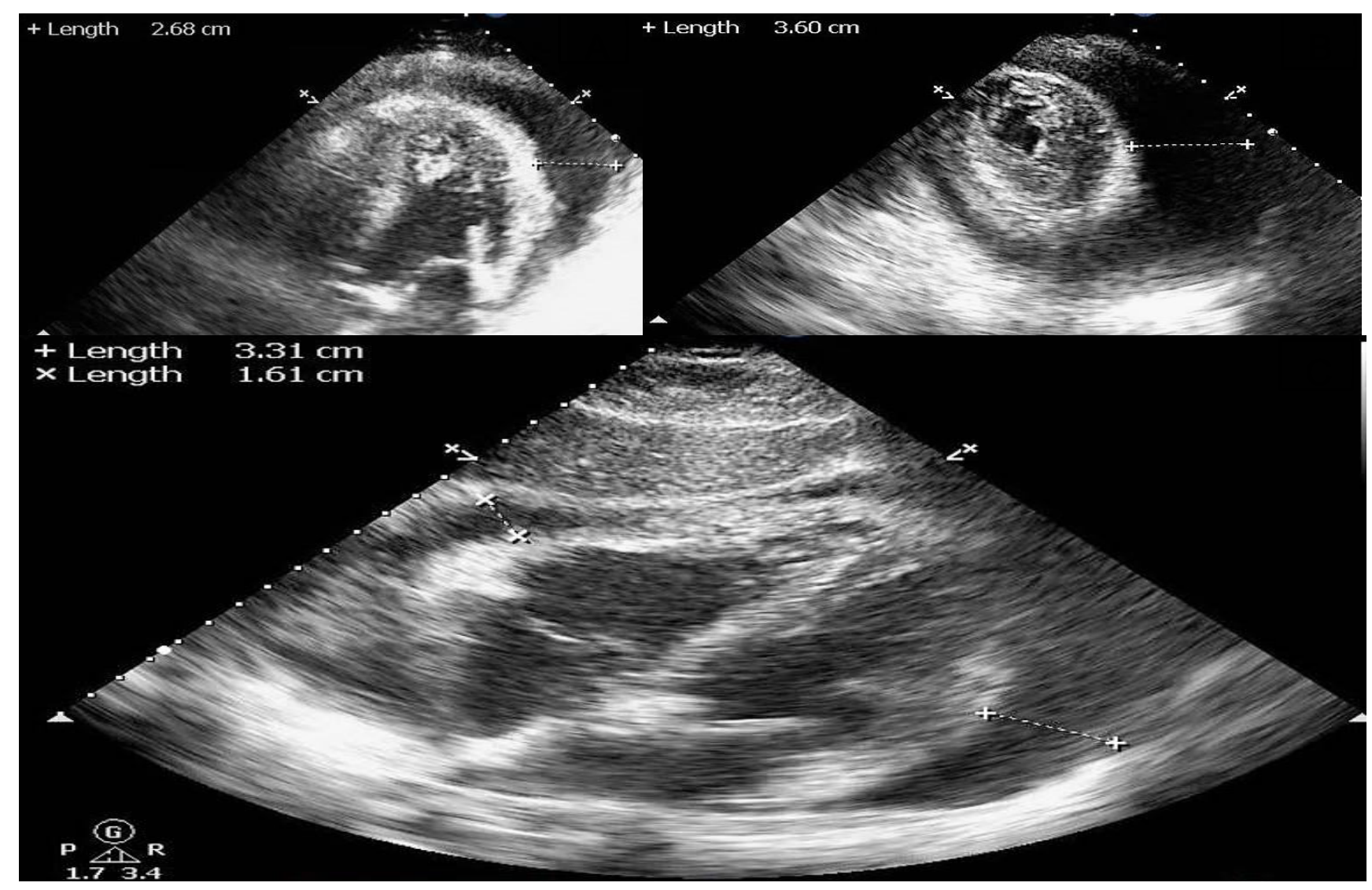

Figure 1: Echocardiogram demonstrating the large circumferential pericardial effusion in A) Apical four chamber view; B) Parasternal short axis view; C) Subcostal four chamber view.

sus paradoxus. His BMI was 24.4. Initial laboratory investigations were significant for leukocytosis 15.2 cells/ microL with predominant neutrophils, ESR $77 \mathrm{~mm} / \mathrm{hr}$, CRP $293 \mathrm{mg} / \mathrm{L}, \mathrm{Cr} 2.1 \mathrm{mg} / \mathrm{dL}$ (baseline $1.1 \mathrm{mg} / \mathrm{dL}$ ), INR 2.7, aPTT of $40 \mathrm{sec}$, and mild transaminitis. BNP was 462 $\mathrm{pg} / \mathrm{ml}$ and troponins were unremarkable. CXR revealed bibasilar infiltrates with small bilateral pleural effusions. Electrocardiogram (ECG) showed sinus tachycardia and diffuse ST elevations consistent with acute pericarditis. ECHO revealed moderately reduced systolic function with an ejection fraction (EF) of $40 \%$, a large free-flowing pericardial effusion circumferential to the heart, and doppler evidence suggestive of increased intra-pericardial pressure consistent with early tamponade physiology (Figure 1). The patient was started on prednisone and colchicine for treatment of acute pericarditis and his rivaroxaban was held. He underwent pericardiocentesis and $250 \mathrm{cc}$ of sanguineous fluid was drained. Additional $100 \mathrm{cc}$ of output through pericardial drain was noted after several hours post-procedure with minimal drain output thereafter. The pericardial fluid analysis revealed WBC 14,940/microL (94\% polymorphonuclear), RBC 545,000/micro, no organisms on gram stain and culture, and a negative cytology for malignancy. Repeat $\mathrm{ECHO}$ one day later showed decrease in the size of the effusion with no evidence of tamponade. The patient also reported significant symptomatic improvement. He also underwent $\mathrm{CT}$ of the abdomen and pelvis which was negative for any suspicious neoplasm. Ultimately, the patient was discharged on colchicine and prednisone. Anticoagulation was held on discharge as the patient had already completed a six month course of anticoagulation for his provoked DVT with repeat negative doppler ultrasound results. Follow up ECHO one month post discharge revealed complete resolution of his pericardial effusion.

\section{Case 2}

A 65-year-old female from Ghana origin with a past medical history of non-ischemic cardiomyopathy and an EF of $25 \%$, hypertension, type $2 \mathrm{DM}$, chronic atrial fibrillation, and history of left atrial appendage thrombus (on rivaroxaban for $>2$-years) presented with complaints of dyspnea and chest pressure. The dyspnea started two weeks prior to presentation, was non-exertional, and accompanied by chest pressure. Her medication history was significant for rivaroxaban $20 \mathrm{mg}$ daily, metoprolol, losartan, sitagliptin, metformin, and atorvastatin.

Her vital signs were unremarkable except for hypertension. Pertinent physical exam findings included jugular venous distention, irregularly irregular pulse, absence of pulsus paradoxus and normal heart sounds. Her BMI was 30. Initial CBC and BMP were unremarkable with normal kidney and liver functions. Her troponins were normal, and BNP was elevated at $1570 \mathrm{pg} / \mathrm{mL}$. Her INR was 1.4 and a PTT was 31 seconds. CXR showed stable cardiomegaly. Her ECG revealed atrial fibrillation 


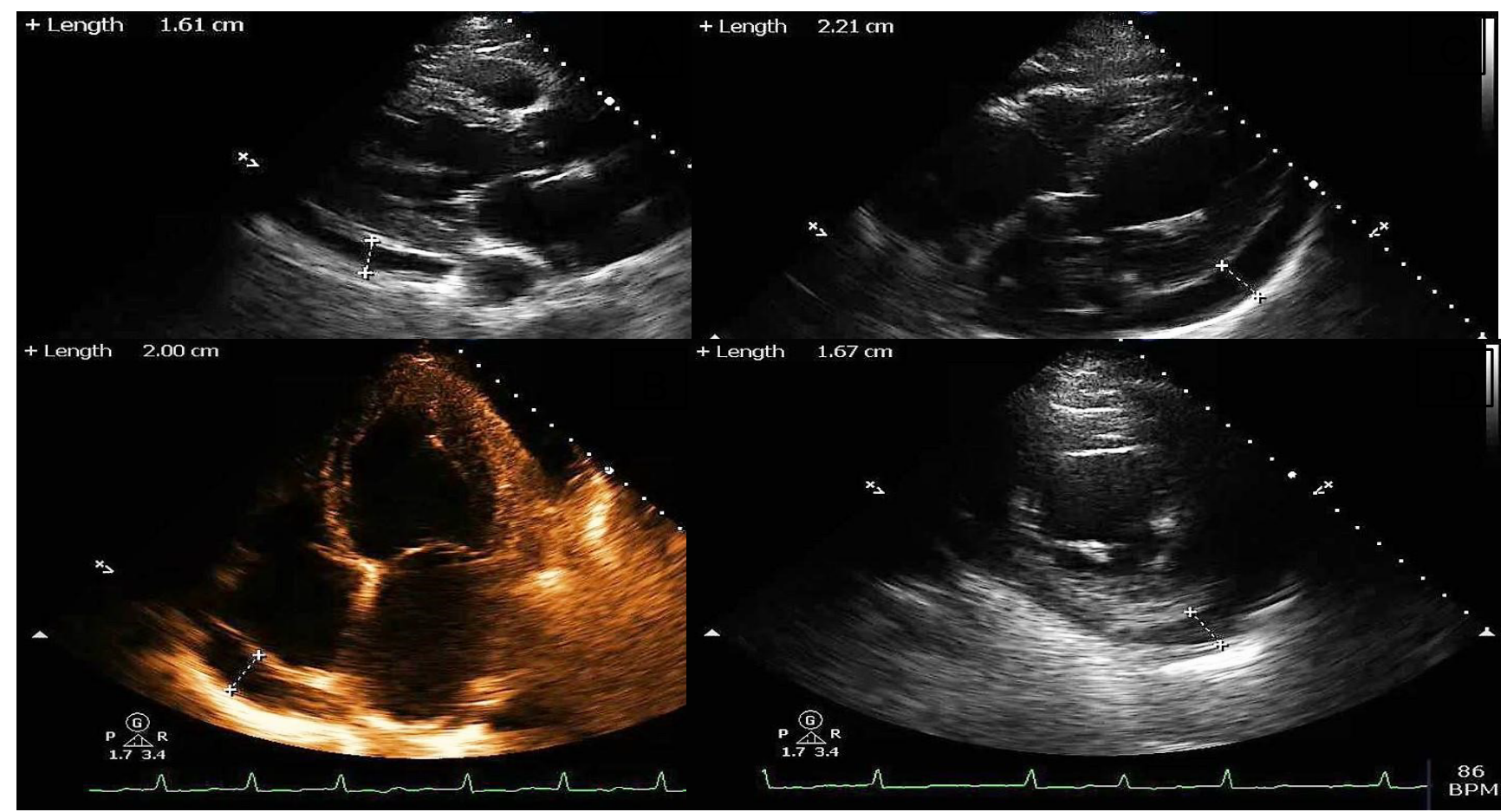

Figure 2: Echocardiogram demonstrating the circumferential pericardial effusion in A) Parasternal long axis view; B) Apical four chamber view; C) Subcostal four chamber view; D) Parasternal short axis view.

with new inverted T waves in the inferolateral leads. On day two, ECHO was performed which revealed moderate to large pericardial effusion with maximum posterior space of $2.21 \mathrm{~cm}$ and anterior space of $2.0 \mathrm{~cm}$ (Figure 2). A diastolic RV (but not RA) collapse was noted and doppler pattern was not suggestive of a tamponade. EF was noted to be $10-15 \%$. Pericardiocentesis was done which revealed $700 \mathrm{cc}$ of bloody fluid material. The fluid analysis revealed 28090000/microL RBC and 1500/ microL WBC (95\% mononuclear). The gram stain and culture revealed no growth and cytology did not reveal any malignant cells. Pericardial drain output was noted to be $400 \mathrm{cc}$ within the first 24 hours post-procedure with minimal output thereafter. Serial echocardiograms revealed significant improvement in the size of the effusion without any evidence of re-accumulation. Patient also reported significant symptomatic improvement. CT chest and abdomen was done which was unremarkable for underlying malignancy.

On discharge, her rivaroxaban was discontinued. ECHO at time of discharge revealed EF of $15-20 \%$ with a small predominantly posterior pericardial effusion. She was followed up as an outpatient after one month and reported no further complaints. Follow up ECHO two months post discharge revealed improvement in the EF to $35 \%$, with a trivial pericardial effusion posteriorly.

\section{Case 3}

A 77-year-old Caucasian female with a past medical history of paroxysmal atrial fibrillation, COPD, significant CAD with multiple stenting of right coronary artery (last one was five months ago prior to presentation) presented with acute onset of shortness of breath and fatigue for two days. Her past medical history was also significant for renal cancer $s / p$ right sided nephrectomy, sub-centimeter lung nodules, and cervical cancer $s / p$ total abdominal hysterectomy. Her medications included rivaroxaban $7.5 \mathrm{mg}$ daily (she was started on low dose rivaroxaban due to being underweight and episodes of bruising while on full dose of anticoagulation), clopidogrel, lisinopril, amlodipine, carvedilol, tramadol, lorazepam PRN, vicodin PRN and famotidine. Upon arrival, she was hemodynamically unstable with persistent hypotension. She was minimally responsive to fluids and required vasopressor support. Her physical exam was pertinent for distant heart sounds, negative jugular venous distention, and clear lungs. Pulsus paradoxus was not assessed. Her BMI was 16. Initial blood work revealed $\mathrm{Hb}$ of $8.1 \mathrm{~g} / \mathrm{dl}$, leukocytosis $12.5 \mathrm{~K} / \mathrm{microL}$ with predominant neutrophils, acute renal failure with $\mathrm{Cr}$. of $2.2 \mathrm{mg} / \mathrm{dl}$ (baseline $0.6 \mathrm{mg} / \mathrm{dl}$ ), CRP $64.2 \mathrm{mg} / \mathrm{l}$, and negative troponins. Coagulation profile was unfortunately not obtained on admission. She had a CXR which revealed enlarged cardiac silhouette which was a significant change compared to a CXR done 2 weeks prior. Point of care ultrasound was obtained at the bedside which revealed a large circumferential effusion (Figure 3). The patient was managed as a case of cardiac tamponade and was taken to the cardiac catheterization lab where she underwent emergent pericardiocentesis with drainage of a substantial amount of hemorrhagic fluid (the amount was not documented). Unfortunately, the procedure was complicated by right ventricular puncture with about 500-600 cc of blood loss and subsequent pulseless ventricular tachycardia requiring 2 minutes of CPR with ROSC. The patient required endo- 


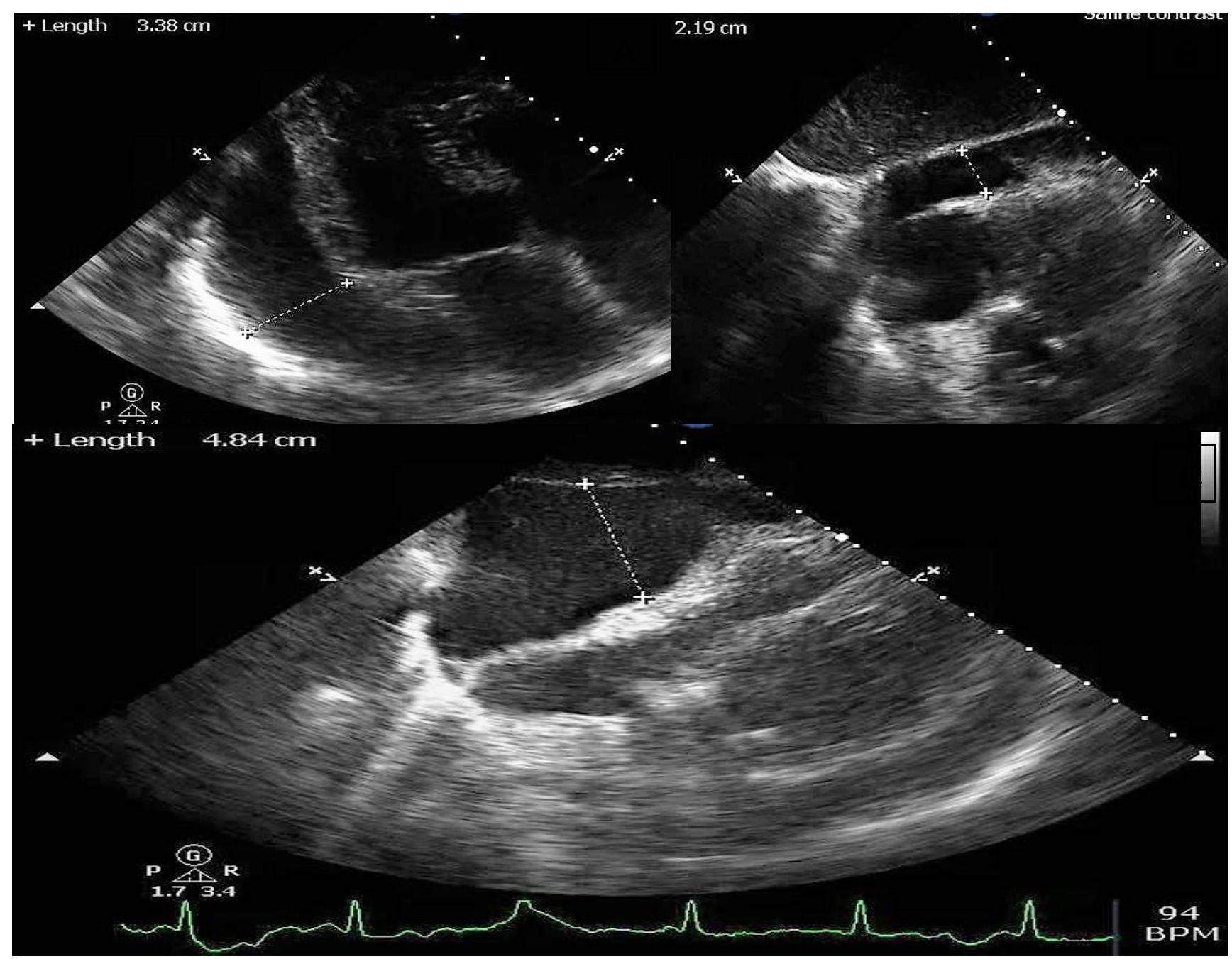

Figure 3: Echocardiogram demonstrating the large circumferential pericardial effusion in A) Off axis parasternal long axis view; $B, C)$ Subcostal four chamber view during inspiration and expiration.

tracheal intubation and was transfused with four units of packed RBCs. A pericardial drain was placed, and the drain output was $650 \mathrm{cc}$ of hemorrhagic fluid within the first 24 hours with minimal drain out thereafter. Serial ECHO did not show re-accumulation of pericardial fluid. The pericardial fluid analysis revealed RBC 315,000/ microL, WBC 629/microL (89\% polymorphonuclear). Cytology revealed acute inflammation with blood, but no malignant cells were seen. Gram stain and culture did not reveal any growth. Patient was extubated after 48 hours and dual antiplatelet therapy was started. Rivaroxaban was held. Her ECHO prior to discharge revealed normal left ventricular function and resolution of pericardial effusion.

\section{Discussion}

Hemopericardium is most often attributed to malignancy, tuberculosis, percutaneous intervention procedures, complication of myocardial infarction, idiopathic and uremia [10]. Novel oral anticoagulants are rarely considered to be in the differentials. It appears that all our patients experienced spontaneous hemopericardium while taking rivaroxaban as a thorough workup excluded other major etiologies.
Patients in case 1 and 2 were taking full strength rivaroxaban $20 \mathrm{mg}$ daily with duration of more than nine months and two years respectively. However, the patient in case 3 was on low dose rivaroxaban 7.5 $\mathrm{mg}$ daily for five months. Only case 3 was on concomitant antiplatelet agent i.e. clopidogrel. In addition, it should be noted that case 1 had concomitant pericarditis and underwent an uncomplicated cardiac catheterization without $\mathrm{PCl}$ one week ago. The cardiac catheterization for case 3 was done five months ago during which two drug eluting stents were deployed. We do not think that there was a traumatic tap in case 3 and procedural complication occurred after pericardiocentesis was performed.

Rivaroxaban has been FDA approved since 2011. It is popular among clinicians due to lack of frequent blood monitoring, once daily dosing, and fewer drug interactions. With its widespread use, there have been multiple case reports of unusual bleeding events including hemopericardium, which were not identified in meta-analyses and post-marketing experience $[1,2]$. To our knowledge, the first case of spontaneous hemopericardium was reported in 2014 [3], and since then few other cases have been reported 
[5-9]. There are two case reports of rivaroxaban induced hemopericardium in setting of acute pericarditis which were similar to our case $1[4,5]$. None of the published case reports in the literature have recent cardiac catheterization done as seen in our first case.

Review of these published case reports also highlight various risk factors that could have contributed in the development of hemopericardium. Concomitant use of saw palmetto and in vitro inhibitor of $\mathrm{CY}$ P3A4 have been implicated in the case report published by Shivamurthy, et al. [3]. Renal insufficiency and simultaneous use of azithromycin was attributed by Boone S in his case report [4]. Menedez, et al. suspected co-administration of multiple drugs metabolized by CYPA34 (including omeprazole, atorvastatin, dronedarone, tamsulosin, and tadalafil) as the causative factor for hemopericardium [7]. Similarly, renal insufficiency and concurrent use of amiodarone (an inhibitor of CYP3A4 and P-glycoprotein) was considered by Oladiran, et al. [9]. Also, the combined use of aspirin and rivaroxaban was seen in only two reported cases $[4,5]$. Compared to these published cases, our case series has identified acute renal insufficiency, concomitant use of clopidogrel and rivaroxaban, and recent cardiac catheterization as potential risk factors. The presence of CYP3A4 substrates were seen in case 2 (atorvastatin) and case 3 (lorazepam, vicodin and tramadol). However, it should be noted that in case 2 there was no report of multiple CYP3A4 drugs other than atorvastatin, and use of opiates and benzodiazepine was only as PRN in case 3 . Thus, it is unclear if this mechanism has played a significant role in our case series.

Other anticoagulants including warfarin, apixaban and dabigatran have also been associated with hemopericardium $[3,11,12]$. Considering the frequent use of these novel anticoagulants and possible association with spontaneous hemopericardium as mentioned in our case series and other published case reports, an early ECHO seems to be a reasonable test for evaluation of pericardial effusion in a patient presenting with chest pain and dyspnea while on these agents in an appropriate clinical setting. Prescribers should be attentive to various factors as mentioned above that can enhance the anticoagulant effects of these agents and lead to spontaneous hemorrhage. As of 2018, andexanet alfa has been FDA approved for reversal of factor Xa inhibitors in a major life-threatening hemorrhage associated with anti-factor Xa medications [13], however, its limited availability and lack of consensus guidelines limits its widespread use. In conclusion, we present a case series of hemorrhagic pericardial effusion likely related to rivaroxaban use. Clinicians should be aware about this potential adverse effect of rivaroxaban and consider hemorrhagic pericardial effusion as a differential in patient presenting with dyspnea and chest pain in an ap- propriate clinical setting. Early recognition by an echocardiogram and prompt treatment can prevent serious life-threatening complications.

\section{Conflict of Interest}

The authors declare that there is no conflict of interest regarding the publication of this article.

\section{Authors Contribution}

All authors have contributed equally to this manuscript.

\section{References}

1. Wasserlauf G, Grandi SM, Filion KB, Eisenberg MJ (2013) Meta-analysis of rivaroxaban and bleeding risk. Am J Cardiol 112: 454-460.

2. (2011) Xarelto (rivaroxaban). Janssen Pharmaceuticals, Titusville, NJ, USA.

3. Shivamurthy P, Brar N, Therrien ML (2014) Isolated hemopericardium associated with rivaroxaban: First case report. Pharmacotherapy 34: e169-e172.

4. Boone S (2015) Cardiac tamponade associated with rivaroxaban. Del Med J 87: 206-207.

5. Xu B, A Maclsaac (2014) Life-threatening haemorrhagic pericarditis associated with rivaroxaban. Int $\mathrm{J}$ Cardiol 174: e75-e76.

6. Basnet S, Tachamo N, Tharu B, Dhital R, Ghimire S, et al (2017) Life-threatening hemopericardium associated with rivaroxaban. Case Rep Cardiol 2017.

7. Menendez D, J Michel (2016) Hemopericardium with tamponade following rivaroxaban administration and its attenuation by CYP3A4 inhibitors. Proc (Bayl Univ Med Cent) 29: 414-415.

8. Kham NM, M Song (2016) Spontaneous, life-threatening hemorrhagic cardiac tamponade secondary to rivaroxaban. Am J Ther 23: e1128-e1131.

9. Oladiran O, Segal J, Nwosu I, Nazir S (2018) A rare case of spontaneous cardiac tamponade induced by concomitant use of rivaroxaban and amiodarone. Case Rep Cardiol 2018.

10. Atar S, Chiu J, Forrester JS, Siegel RJ (1999) Bloody pericardial effusion in patients with cardiac tamponade: Is the cause cancerous, tuberculous, or iatrogenic in the 1990s? Chest 116: 1564-1569.

11. Sigawy C, Apter S, Vine J, Grossman E (2015) Spontaneous hemopericardium in a patient receiving apixaban therapy: First case report. Pharmacotherapy 35: e115-e117.

12. Lee KS, T Marwick (1993) Hemopericardium and cardiac tamponade associated with warfarin therapy. Cleve Clin $\mathrm{J}$ Med 60: 336-338.

13. Heo YA (2018) Andexanet alfa: First global approval. Drugs 78: 1049-1055. 